\title{
New Radio and Optical Data for GRO J1655-40
}

\author{
Richard W. Hunstead, Kinwah Wu, Duncan Campbell-Wilson \\ School of Physics, University of Sydney, NSW 2006, Australia
}

\begin{abstract}
.
MOST $843 \mathrm{MHz}$ flux densities are presented for the May 1996 outburst from GRO J1655-40. A deep radio image of the field reveals extended emission regions which may be associated with the radio jets. The optical spectrum during the 1994 outburst shows remarkable similarities to that of a Wolf-Rayet WN star.
\end{abstract}

\section{Introduction}

Radio emission from the extraordinary black hole X-ray binary GRO J1655-40 was first detected in August 1994 by the Molonglo Observatory Synthesis Telescope (MOST) at $843 \mathrm{MHz}$ (Hunstead et al. 1995). A detailed light curve, spanning the three main radio outbursts in 1994, is discussed by Wu and Hunstead (this volume). In addition to the radio data, high-resolution optical spectra of GRO J1655-40 were obtained at the Anglo-Australian Telescope (AAT) in August-September 1994 during the decline from the first radio outburst.

After a quiescent period of 18 months, during which there were several hard $\mathrm{X}$-ray outbursts, GRO J1655-40 returned briefly as a radio source in May-June 1996 (Hunstead \& Campbell-Wilson 1996). Here we present a preliminary interpretation of this recent radio outburst and discuss some interesting properties of the optical spectrum at a time when the light output was dominated by the accretion disk.

\section{Radio Observations}

The MOST (Robertson 1991) is an east-west synthesis telescope operating at $843 \mathrm{MHz}$ with a resolution of $43^{\prime \prime} \times 43^{\prime \prime} \operatorname{cosec}(\delta)$ and a field of view of $23-160^{\prime}$, selectable via time-shared beam switching. The rms noise in a 12-hour synthesis is typically $<1 \mathrm{mJy}$ for the $23^{\prime}$ field used in our X-ray binary monitoring program.

The MOST light curve for the May 1996 event in GRO J1655-40 is shown in Fig. 1. Each point records the mean flux density (and formal error) of a 12-hour observation, measured on a CLEANed image by the AIPS task IMFIT. Subdivision of each 12-hour integration reveals significant variations on shorter timescales.

The first four points in Fig. 1 define an approximately exponential decay with an e-folding time of 1.4 days, similar to the decay rates seen for the shortlived events in 1994. However, the later points, although of lower significance, 


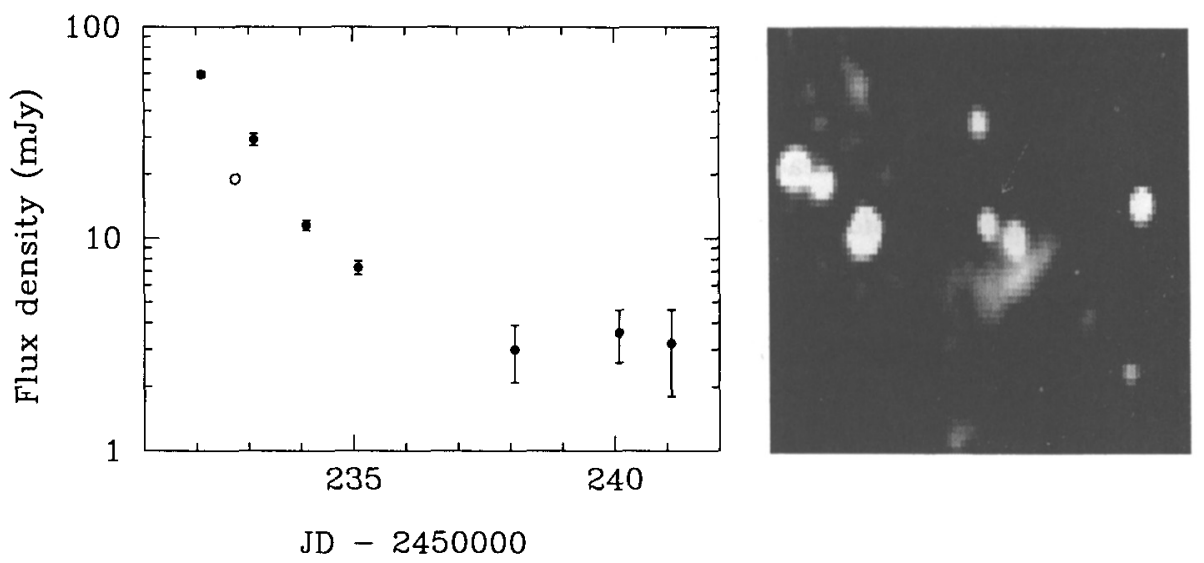

Figure 1. Left: MOST $843 \mathrm{MHz}$ light curve for the May 1996 outburst; the VLA $4.8 \mathrm{GHz}$ measurement is shown as an open circle. Right: Sum of eight MOST images of the field; the area shown is $18^{\prime} \times 18^{\prime}$. North is up and east to the left. The mean flux density of GRO J1655-40 (arrowed) in this montage is $10 \mathrm{mJy}$ and the rms noise is $0.3 \mathrm{mJy} /$ beam; the faintest grey levels correspond to $\sim 2.5$ mJy/beam.

fall well above an extrapolation of the exponential and may signify either an underlying radio component or a second, much weaker outburst. The only other detection in this period, with the VLA at $4.8 \mathrm{GHz}$ (Hjellming \& Rupen 1996), yields a 2-point spectral index $\alpha \simeq 0.4\left(S \propto \nu^{-\alpha}\right)$; this is also consistent with the short-lived 1994 events. It is important to note that there was no obvious hard X-ray precursor to the May 1996 radio event although the source was detected by BATSE after the event (C. Robinson, priv comm). However, there was a soft X-ray turn-on earlier in the month (Remillard et al. 1996) and a progressive hardening of the spectrum thereafter.

Also shown in Fig. 1 is a montage of eight images of the radio field from May, June and July 1996, including observations made before, during and after the outburst. This image shows two regions of extended low-surface-brightness emission aligned approximately with the p.a. $\left(47^{\circ}\right.$ ) of the radio jets (Hjellming $\&$ Rupen 1995). If the association is real this system bears a remarkable resemblance to the classic triple structure seen on $\mathrm{kpc}-\mathrm{Mpc}$ scales in quasars and radio galaxies. Alternatively we may be seeing a remnant from the formation of the black hole. At an assumed distance of $3.5 \mathrm{kpc}$ the closer and brighter of the putative lobes is at a projected separation of $2.5 \mathrm{pc}$ from the binary.

\section{Optical observations}

Short-exposure optical spectra of GRO J1655-40 were obtained with the 3.9-m AAT and RGO spectrograph over the period 1994 Aug 26-Sep 6. We concentrate here on the six nights Aug 30-Sep 4 (TJD 9594.4-9599.4) in which good S/N 
spectra were obtained in two spectral regions, one centred at $\mathrm{H} \alpha$ and the other at $\mathrm{He} \mathrm{II} / \mathrm{H} \beta$. Exposure times were typically $1000-1200 \mathrm{~s}$ in each region. Resolution was $1.3 \AA \mathrm{FWHM}$, corresponding to $58 \mathrm{~km} \mathrm{~s}^{-1}$ at $\mathrm{H} \alpha$ and $85 \mathrm{~km} \mathrm{~s}^{-1}$ at He II.

Significant changes were observed from night to night in the structure, wavelength centroid and equivalent width of the prominent emission lines, all of which were well resolved. A full discussion of the spectra will be given elsewhere. For the time being, we concentrate on the summed spectra in each region, noting that a uniform sampling of binary phase was achieved over the six nights.

The strongest lines, $\mathrm{H} \alpha$ and He II, showed clear double structure on half the nights, and incipient double structure on the remainder. The natural interpretation of such a profile is that it arises from material in the approaching and receding edges of an accretion disk. From the period and masses determined by Bailyn et al. (1995) for this system, we derive an orbit radius of $\sim 10^{7} \mathrm{~km}$ and a Keplerian velocity of $\sim 250 \mathrm{~km} \mathrm{~s}^{-1}$. This then sets a lower limit of $>500 \mathrm{~km} \mathrm{~s}^{-1}$ on the separation of the peaks if they have a disk origin. In fact the separation is always less than $500 \mathrm{~km} \mathrm{~s}^{-1}$, being greatest at $350 \mathrm{~km} \mathrm{~s}^{-1}$ on Sep 1; we conclude, therefore, that the Balmer lines and He II are unlikely to originate solely in the accretion disk.

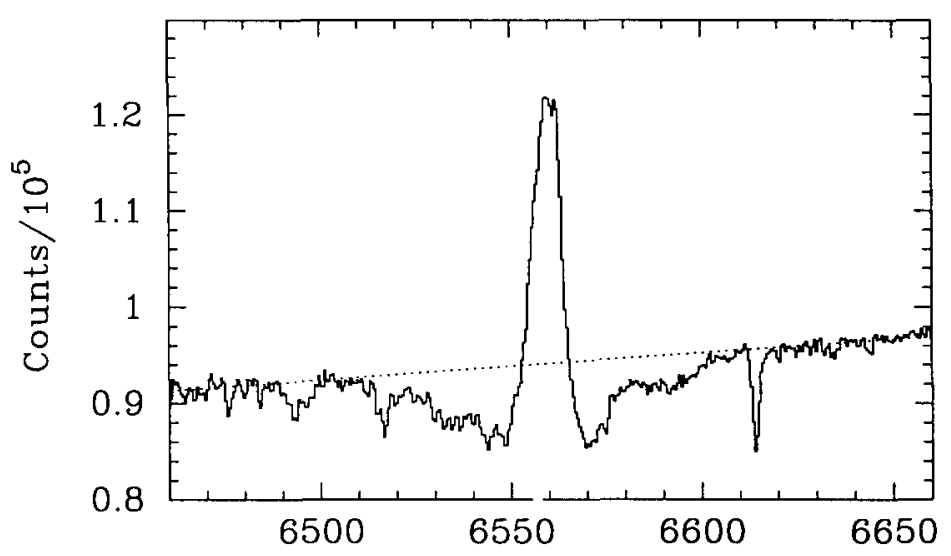

Wavelength $(\AA)$

Figure 2. Summed spectrum of GRO J1655-40 in the region of $\mathrm{H} \alpha$; the dotted line marks the approximate continuum level. The absorption line at $6613 \AA$ is interstellar.

The summed spectrum near $\mathrm{H} \alpha$ is shown in Fig. 2. The FWHM of the emission line is $400 \mathrm{~km} \mathrm{~s}^{-1}$ but it sits inside an absorption bowl with a total extent of $\sim 4000 \mathrm{~km} \mathrm{~s}^{-1}$. This broad, structured absorption trough probably arises in an expanding corona which envelopes the accretion disk.

Fig. 3 shows the co-added He II/H $\beta$ spectrum together with a composite Wolf-Rayet WN spectrum from the atlas compiled by Hamann et al. (1995). The WN composite consists of the merged sum of WR 82 (spectral type WN8), WR 91 (WN6-s, formerly WN7) and WR 100 (WN6-s), chosen to provide a 


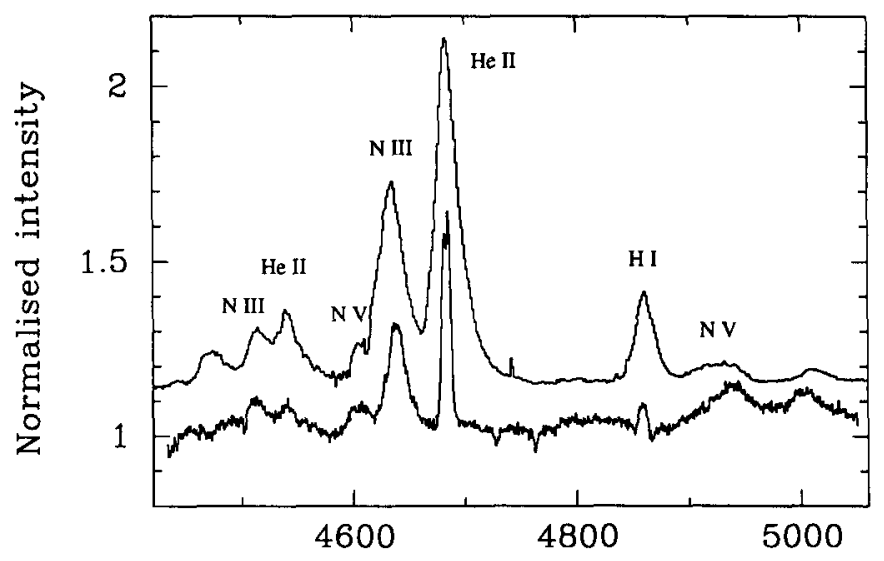

Wavelength $(\AA)$

Figure 3. Lower: Summed spectrum of GRO J1655-40 in the region of $\mathrm{H} \beta$ and $\mathrm{He}$ II, normalised to the continuum. Upper: Composite WolfRayet WN spectrum from Hamann et al. (1995), scaled and offset in zero-point. The most prominent emission lines are marked.

reasonable match to the relative strengths of features in the spectrum of GRO $\mathrm{J} 1655-40$.

The similarity between the two spectra in Fig. 3 is particularly striking, especially for the broad high-excitation lines, suggesting that the conditions of temperature and outflow in an accretion disk corona mimic closely those in W-R stars of spectral class WN6-8. It is worth noting that the W-R features disappeared at the onset of the second X-ray outburst on Sep 6 .

A further comparison was made between GRO J1655-40 and the published spectrum of another black-hole binary V404 Cyg (Casares et al. 1991; Fig. 3). Once again there was remarkable correspondence, especially for the broad W-R features that might otherwise have gone unnoticed. Finally, there is the interesting case of Cyg X-3 in which IR spectra have been interpreted as suggesting that the companion is a Wolf-Rayet star of spectral type WN7 (van Kerkwijk et al. 1992). On the basis of our findings for GRO J1655-40 and V404 Cyg we speculate that Cyg X-3 may also be a black-hole LMXB, with the W-R characteristics being associated with the hot, dense region of the inner disk corona rather than a companion star.

We thank our colleagues for allowing the encroachment of GRO J1655-40 observations on their scheduled AAT programs, and the staff at the Molonglo Observatory for their heroic efforts in completing the mechanical repair that enabled our discovery of the May 1996 outburst. The MOST is supported by grants from the Australian Research Council and the Science Foundation for Physics within the University of Sydney. 


\section{References}

Bailyn, C. D. et al. 1995, Nature, 374, 701.

Casares, J. et al. 1991, MNRAS, 250, 712.

Hamann, W.-R. et al. 1995, A\&AS, 113, 459.

Hjellming, R. M. and Rupen, M. P. 1995, Nature, 375, 8

Hjellming, R. M. and Rupen, M. P. 1996, IAU Circ 6411.

Hunstead, R. W. et al. 1995, Compact Stars in Binaries, eds. J. van Paradijs et al., Dordrecht: Kluwer, p.369

Hunstead, R. and Campbell-Wilson, D. 1996, IAU Circ 6410.

van Kerkwijk, M. H. et al. 1992, Nature, 355, 703.

Remillard, R. et al. 1996, IAU Circ 6393.

Robertson, J.G. 1991, Aust J Phys, 44, 729.

\section{Discussion}

D. Meier: What are the velocity widths of the emission lines in the Wolf-Rayet state?

R. Hunstead: Approximately $500 \mathrm{~km} \mathrm{~s}^{-1}$. Note that this will appear in the written version of the paper. 